\title{
Support Vector Machines for Multi-Attribute ABC Analysis
}

\author{
Hasan Basri Kartal and Ferhan Cebi
}

\begin{abstract}
This paper examined the classification performance of Support Vector Machines (SVMs) on multi-criteria inventory analysis. The $\mathrm{ABC}$ analysis using the Simple Additive Weighting (SAW) method was employed to determine inventory classes of items held in inventory of a large scale automobile company operating in Turkey. The provided data set was analyzed with SVMs to obtain classification performance of the SVM learning algorithm. The results showed that SVM is highly applicable to the inventory classification problem.
\end{abstract}

Index Terms-ABC analysis, multi-criteria inventory classification, support vector machine (SVM).

\section{INTRODUCTION}

Inventory management is a crucial issue requiring managerial concerns because of its effects on operational performance and competitiveness of organizations. Organizations need managerial tools in analyzing inventory efficiently. $\mathrm{ABC}$ analysis is one of the most commonly used methods to classify items held in inventory with regard to their relative importance. This kind of classification provides the organizations determine the most important items in inventory which the organizational resources will focus on [1].

$\mathrm{ABC}$ analysis classifies inventory items into three different groups as $\mathrm{A}, \mathrm{B}$, and $\mathrm{C}$ by their relative significance based on well-known Pareto principle. In conventional ABC Group A items are relatively few in number of unites but consist of the large amount of annual usage dollar value, whereas Group $C$ items are relatively large in number of units but consists few of the amount of annual usage [2]. The remaining items between the two groups are Group B. Traditional ABC analysis is commonly applied to split inventory items into groups because of its simplicity and ease of use. However, the analysis has been criticized because it considers only a single dimension, the total amount of usage cost [3]-[5]. Thus, in many cases that other attributes can also be important, classical $\mathrm{ABC}$ inventory analysis becomes insufficient. Hence, multi-criteria inventory classification provides an opportunity to consider additional criteria such as criticality, lead time, substitutability, commonality, repairability, storage cost, scarcity cost, payment options, supplier alternative, unite size, order size, or such others [6], [7]. Therefore, multi-criteria $\mathrm{ABC}$ analyses have been getting

Manuscript received December 08, 2012; revised January 15, 2013.

The authors are with Faculty of Management, Istanbul Technical University, Istanbul, Turkey (e-mail: basrikartal@gmail.com; cebife@itu.edu.tr). attractions for more than two decades.

There are many multi-criteria $\mathrm{ABC}$ analysis models in the literature such as matrix-based methodologies, analytical hierarchy process (AHP) based and linear optimization based models, and meta-heuristic approaches. A joint criteria matrix approach applied via bi-criteria as in [2], [8] can be given as examples of matrix-based models. Reference [2] extended a previous study and suggested the use of AHP to integrate the given multiple criteria and to rank the items in inventory. Reference [8], [9] used an AHP based model to classify inventory items. Unit cost, annual dollar usage and several additional criteria such as critical factor and lead time were considered in the classification of items in [9]. Reference [10] applied a fuzzy AHP based model for classifying the inventory items to be hold in a distributing firm. In the study [10], fuzzy theory was used to overcome the difficulty on determining the importance of the conflicting attributes. Reference [11] proposed another approach named as $A B C-F C$ approach by combining $\mathrm{ABC}$ analysis and fuzzy theory for classification of items.

Data envelopment analysis (DEA) based models such as weighted linear optimization [7], [12] were also used in $\mathrm{ABC}$ inventory classification by considering multiple criteria. Reference [13] proposed a modified version of a common weight DEA-like model by applying a few concepts in DEA models to use linguistic terms in the model. Whereas [7] proposed a weighted linear optimization model which is likely to the method of data envelopment analysis, [12] provided an extended version of a previous model by using two sets of weights as both most favorable and less favorable for each inventory item. The literature also introduced the applications based on heuristic techniques for classifying inventory items based on multiple criteria. Reference [14], for example, employed SVM and k-nearest neighbor to classify the inventory items. Reference [15] proposed an artificial neural network (ANN) to address the problem of inventory classification of a company in a pharmaceutical industry. Reference [3] used the genetic algorithm to find a solution to multi-attribute classification problem by optimization a set of parameters representing the weights of criteria.

The purpose of this paper is to investigate SVMs performance in inventory classification based on multiple attributes. The remaining of the paper is organized as follows. The next section gives brief information on SVMs. Section III introduces a real case study of an automobile company as an application; Section IV discusses the results of the application. Finally, the last section presents the conclusion section based on the findings. 


\section{Support Vector Machines}

SVMs are a set of related machine learning methods based on Vladimir Vapnik's statistical learning theory. A SVM classifier takes a set of inputs which belong to different classes, builds a model that predicts any given instance belongs to which class [16], [17]. It determines an optimal hyperplane to separate different classes in the data set. The hyperplane is placed at the maximum distance from the nearest points of a given data set [18].

A SVM model represents the training examples into separated categories in a mapped space as certain points [17]. In this space, finding the hyperplane requires to solve a quadratic optimization problem by using Lagrange multipliers [18]. The points, which determine the hyperplane, are called Support Vectors. Thus, the vectors are critical elements to train the classifying algorithm. It determines an optimal hyperplane to separate different classes in a data set [19].

As $m$ labels the given training examples in a given set where $x_{i}$ is the feature of the $i$ th example, $y_{i}$ defines the output for $i$ th example as a binary value, and $\mathrm{w}$ denotes the weight and $b$ the bias in (1) and (2).

$$
\begin{aligned}
& w X_{i}+b \geq+1 \text { for } y_{i}=+1 \\
& w X_{i}+b \leq-1 \text { for } y_{i}=-1
\end{aligned}
$$

If these conditions in (1) and (2) are considered for each pairs of $\left(x_{i}, y_{i}\right)$ while $i=1,2 \ldots m$ we can form it as in (3).

$$
\left\{\left(x_{i}, y_{i}\right) \mid x_{i} \in R^{N}, y_{i} \in\{-1,1\}\right\}_{i=1}^{m}
$$

In order to determine the hyperplane which was established as far from the support vectors as possible, the margin supposed to be maximized. Thus maximization of the margin is equivalent to minimization of $\|w\|$. We may get the minimum $\|w\|$ by quadratic programing as shown below.

$$
\begin{gathered}
\min \frac{1}{2} w^{T} w \\
\text { s.t. } y_{i}\left(w^{T} x_{i}+b\right) \geq 1 \text { and } i=1,2, \ldots, m
\end{gathered}
$$

This quadratic problem can be solved by introducing Lagrange multipliers using the Karush-Kuhn-Tucker theory. $\mathrm{C}$ in (7) is a penalty parameter [20].

$$
\begin{gathered}
\max \sum_{i=1}^{m} \alpha_{i}-\frac{1}{2} \sum_{i=1}^{m} \sum_{i=1}^{m} \alpha_{i} \alpha_{j} y_{i} y_{j} x_{i}^{T} x_{j} \\
\text { s.t. } \sum_{i=1}^{m} \alpha_{i} y_{i}=0,0 \leq \alpha_{i} \leq C \text { and } i=1,2, \ldots, m
\end{gathered}
$$

SVM algorithms use also kernels to reduce the complexity of problems as mapping them in a high dimensional space [21]. A kernel function makes easier to classify the inputs using the kernel trick as follows:

$$
K\left(x_{i}, x_{j}\right)=\varphi\left(x_{i}\right)^{T} \varphi\left(x_{j}\right)
$$

To find the optimal hyperplane we can reform the problem in a new quadratic model as follows:

$$
\begin{aligned}
& \max \sum_{i=1}^{m} \alpha_{i}-\frac{1}{2} \sum_{i=1}^{m} \sum_{i=1}^{m} \alpha_{i} \alpha_{j} y_{i} y_{j} \varphi\left(x_{i}\right)^{T} \varphi\left(x_{j}\right) \\
& \text { s.t. } \sum_{i=1}^{m} \alpha_{i} y_{i}=0,0 \leq \alpha_{i} \leq C \text { and } i=1,2, . ., m
\end{aligned}
$$

For each of the $\mathrm{n}$ support vector, the decision function $D(x)$ which is not a magnitude but the sign becomes as in (11).

$$
D(x)=\operatorname{sign}\left(\sum_{k=1}^{n} a_{k} y_{k} K\left(x, x_{k}\right)+b\right)
$$

There are various kernel functions used by SVM such as radial basic function, sigmoid function and polynomial function. More information on SVM algorithms and applications can be found in [19], [22] and details of the statistical learning theory are available in [21], [23].

\section{APPLICATION OF SVM ClassificATION}

This study aimed to investigate SVMs' applicability in multi-attribute ABC inventory analysis. First the simple additive weighting (SAW) method was implemented to classify inventory items based on the multiple attributes. Then SVMs were applied for measuring its classification performance.

The multi-attribute inventory classification was applied to 715 industrial inventory items of a large scale automotive company operating in Turkey [24]. The attributes used in the analysis were realized by discussing with the engineers in the related department. These attributes were illustrated in Fig.1.

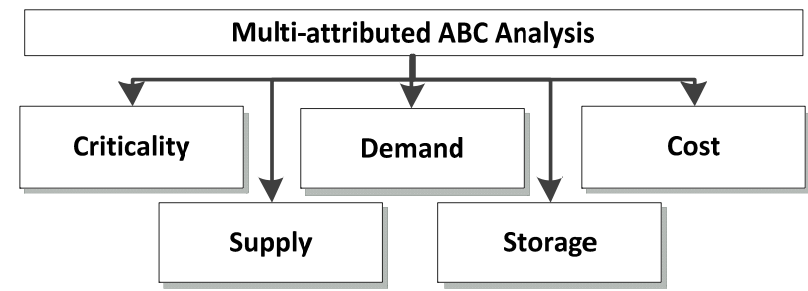

Fig. 1. The attributes of the applied multi-attributed ABC analysis model

Criticality is a factor referring the importance of the inventory items with respect to ongoing and planned production processes [8]. An item with a high level of criticality has more importance to the continuity of the production and to overall manufacturing efficiency.

Demand describes the total number of usage for each item which was consumed in a year [10]. The higher number it is used the higher importance it has.

Cost refers to the unit cost of an inventory item as an amount of money. It is considered as the purchase price of an item to the company [8], [10].

Supply attribute was defined as a value based on the supplying related importance of an inventory item. The inventory in the industrial material warehouse is a group of inventory that consists of items that their being supplied on time is significance, and both the supplier and lead time for an item are important in an adequate supply of an item on time without extra stocking and without excessive costs [25]. The value for the supply attribute is a number between zero and one that was determined by an item's condition of supplier and average lead time in last year's inventory records. Lead time was considered as the time interval 
between ordering and receiving an order. A longer lead time and an unsecure supplier caused a bigger number on supply attribute. A higher value for supply attribute means a higher level of importance in inventory management; and it requires more care to make improvement because the stability of supplier and length of the lead time are important for safety of supply to provide desired service level from storage to the production processes [26], [27].

Storage is the last attribute, and it is crucial for the warehouse due to the limited capacity. It refers to the storing conditions of an item, basically to the volume occupied by an item and its holding costs in the storage [25], [28]. An item which requires a larger space and a higher holding cost gets a higher value for the storage feature. It was determined similarly as a number between zero and one for each item.

The values for the related attributes of each inventory items were listed on an excel sheet as a data set. The data set consists of values which were provided by the company's last year's inventory records and values were recently assigned by warehouse technicians. The SAW method, which is a commonly known and widely used method for multi-attribute decisions, was applied to the inventory. It was chosen as a model to produce the analysis results because it is very easy to apply and simple to evaluate [23]. The weights of each attributes determined by directly interviewing with the engineers of related department as 0.3 for criticality, 0.1 for cost and 0.2 for demand, supply and storage attributes.

The values in data set were normalized by linear normalization to relatively calculate the contribution of each feature to the item's importance. For each inventory item, the normalized values of attributes were weighted by the determined weights. Then as summing the simple weighted values for each item, the overall importance values have been obtained as the final scores shown blow on Table I.

TABLE I: THE EXAMPLES OF DATA AND FINAL SCORES

\begin{tabular}{cccccc}
\multicolumn{7}{c}{ TABLE I: THE EXAMPLES OF DATA AND FINAL SCORES } \\
\hline \hline Cri. & Demand & Cost & Sup. & Sto. & Final Scores \\
\hline 0.184 & 0.3970 & 0.0001 & 0.3210 & 0.31 & 0.21859 \\
0.428 & 0.0154 & 0.0065 & 0.1871 & 0.31 & 0.23071 \\
0.352 & 0.0001 & 0.6990 & 0.1790 & 0.56 & 0.25981 \\
0.402 & 0.4304 & 0.0002 & 0.2403 & 0.31 & 0.27368 \\
0.428 & 0.0004 & 0.0553 & 0.1871 & 0.13 & 0.20297 \\
0.317 & 0.0056 & 0.0018 & 0.2194 & 0.31 & 0.19925 \\
0.335 & 0.2612 & 0.0014 & 0.3290 & 0.56 & 0.30459 \\
0.352 & 0.0001 & 0.1413 & 0.3129 & 0.31 & 0.28130 \\
0.335 & 0.1025 & 0.0016 & 0.3290 & 0.56 & 0.28876 \\
0.335 & 0.0852 & 0.0006 & 0.0500 & 0.31 & 0.18102 \\
\hline \hline
\end{tabular}

After the final scores were calculated and the results were sorted in order of descending as illustrated in Table II, to classify inventory items into groups A, B, and C, Pareto's 80-20 rule [29] applied to the final importance scores. The top $20 \%$ of the inventory were assigned to group A; the bottom $50 \%$ were assigned to group $\mathrm{C}$, and the between $30 \%$ were assigned to group B. Hence, we obtained a data set of inventory that consist the multiple attributes and classes of each inventory items.

Once classes of the items determined by the model, to analyze the data by SVM a popular data mining software WEKA 3.6.8 [30] has been used. It contains various visualization tools and machine learning algorithms including SVM [31]. In order to measure SVM's classification performance, poly kernel SVM and normalized poly kernel SVM algorithms of SMO function implemented in WEKA have been used. Its implementation transforms nominal attributes into binary values and provides results by normalizing the attributes [30], [32]. Required parameters such as complexity and gamma parameter etc. were assigned as default values.

TABLE II: THE RANKING AND CLASSES OF ITEMS

\begin{tabular}{ccccc}
\hline \hline ID & $\begin{array}{c}\text { SAW } \\
\text { score }\end{array}$ & $\begin{array}{c}\text { Cumulative SAW } \\
\text { score }\end{array}$ & $\begin{array}{c}\text { Cumulative \% of } \\
\text { items }\end{array}$ & Class \\
\hline 485 & 0.427662 & 0.427662 & $0.14 \%$ & $\mathrm{~A}$ \\
442 & 0.408145 & 0.835807 & $0.28 \%$ & $\mathrm{~A}$ \\
73 & 0.241266 & 37.230168 & $20.06 \%$ & $\mathrm{~B}$ \\
30 & 0.240499 & 37.470667 & $20.20 \%$ & $\mathrm{~B}$ \\
142 & 0.240303 & 37.710970 & $20.34 \%$ & $\mathrm{~B}$ \\
568 & 0.203067 & 83.865079 & $50.14 \%$ & $\mathrm{C}$ \\
569 & 0.203067 & 84.068147 & $50.28 \%$ & $\mathrm{C}$ \\
377 & 0.111196 & 144.944232 & $99.72 \%$ & $\mathrm{C}$ \\
122 & 0.108239 & 145.052470 & $99.86 \%$ & $\mathrm{C}$ \\
494 & 0.108014 & 145.160484 & $100.00 \%$ & $\mathrm{C}$ \\
\hline \hline
\end{tabular}

\section{RESULTS}

The performance results of SVM have been evaluated by three performance modes as the percentages of correctly classified items. These modes are training set, cross validation, and percentage split test.

First, all of the data has been used both to train the algorithm and to predict the classes. The simple attributes of the items were taken as inputs, and then the classes of each item were predicted. Secondly, inventory items were divided into 11 folders of nearly equal size of instances for crossvalidation. Finally, the data split into two parts as $66.66 \%$ of training set and $33.33 \%$ of test data for percentage split test. The classification performance results by training set, cross-validation, and percentage split test using both the poly kernel SVM and normalized poly kernel SVM are shown in Table III.

\begin{tabular}{lcc}
\multicolumn{3}{c}{ TABLE III: THE RESUlts FOR SVM's ClASSIFICATION PERFORMANCE } \\
\hline \hline Performance mode & Poly kernel SVM & Norm. poly kernel SVM \\
\hline Training set & $90.396 \%$ & $86.299 \%$ \\
Cross-validation & $88.418 \%$ & $84.887 \%$ \\
Percentage split test & $90.456 \%$ & $86.307 \%$ \\
\hline \hline
\end{tabular}

As seen in the first column of the table above, poly kernel SVM approximately $90 \%$ correctly classified the instances for the three tests mentioned above, whereas as seen in the second column of the table above normalized poly kernel SVM about $85 \%$ correctly classified the instances for the same tests. Although the classification performance for each type of test in both columns varies from $84.3 \%$ to $90.4 \%$, reasonable accuracy results obtained for all the tests in the table.

\section{CONCLUSION}

This paper presents a support machine application in multi-attribute $\mathrm{ABC}$ analysis based on the SAW method. In 
the $\mathrm{ABC}$ analysis inventory classes are obtained by considering multiple criteria such as criticality, demand, cost, supply and storage. By employing the raw inventory data as inputs and the produced classes as outputs, the study utilizes SVMs to measure the algorithm's classification performance. The results indicate that SVMs can be successfully applied to inventory classification problems. However, there is a limitation of the study to generalize the results because only one specific application was conducted in the study. In the case of the usage different inventory data set, similar results from SVMs applications in multi-attribute ABC analyses may not be obtained. The other SVM applications can be useful to improve inventory classification performances. For further studies, several kernel SVMs can be used, and their performance can be compared to achieve better results. Similarly, the studies using various ABC inventory analysis models may be conducted.

\section{REFERENCES}

[1] R. B. Chase, F. R. Jacobs, and N.J. Aquilano, Operations Management: For Competitive Advantage, 10th ed. New York: McGrawe-Hill Irwin, 2004.

[2] B. E. Flores and D. C. Whybark, "Implementing multiple criteria ABC analysis," Journal of Operational Research, vol.7, pp. 79-85, 1987.

[3] H. A. Guvenir and E. Erel, "Multi-criteria inventory classification using a genetic algorithm," European Journal of Operational Research, vol. 105, pp. 29-37, Feb. 1998.

[4] D. Radson and A. H. Boyd, "The Pareto principle and rate analysis," Quality Engineering, vol.10, pp. 223-229, 1997.

[5] W. L. Ng, "A simple classifier for multiple criteria ABC analysis," EJOR, vol. 177, pp. 344-353, Jan. 2006.

[6] Y. Chen, W. Kevin, and L. Si-Feng, "A Comparative study on multi-criteria $\mathrm{ABC}$ analysis in inventory management," IEEE, vol. 38, pp. 3416-3421, China, 2008.

[7] R. Ramanathan, "ABC inventory classification with multiple-criteria using weighted linear optimization," Computers and Operations Research, vol. 33, pp. 695-700, 2006

[8] B. E. Flores, D. L. Olson, and V. K. Doria, "Management of multi-criteria inventory classification," Mathematical and Computer Modeling, vol. 16, pp. 71-82, Dec. 1992

[9] F. Y. Partovi and W. E. Hopton, "The analytic hierarchy process as applied to two types of inventory problems," Production and Inventory Management Journal, vol. 35, pp.13-19, 1994.

[10] F. Cebi, C. Kahraman, and B. Bolat, "A multi-attribute ABC classification model using fuzzy AHP," in Computers and Industrial Engineering, 40th International Conference, Awaji, 2010, pp. 1-6.

[11] C. Chu, G. Liang, and C. Liao, "Controlling inventory by combining $\mathrm{ABC}$ analysis and fuzzy classification," Computers \& Industrial Engineering, vol. 55, pp. 841-851, 2008.

[12] P. Zhou and L. Fan, "A Note on multi-criteria ABC inventory classification using weighted linear optimization," EJOR vol. 182, pp. 1488-1491, Nov. 2006.

[13] S. A. Torabi, S. M. Hatefi, and B. S. Pay, "ABC inventory classification in the presence of both quantitative and qualitative criteria," Computers \& Industrial Engineering, vol. 63, pp. 530-537, 2012.

[14] M. Yu, "Multi-criteria $\mathrm{ABC}$ analysis using artificial intelligence based classification techniques," Expert Systems with Applications, vol. 38, pp. 3416-3421, April 2011

[15] F.Y. Partovi and M. Anandarajan, "Classifying inventory using an artificial neural network approach," Computers \& Industrial Engineering, vol. 41, pp. 389-404, Feb. 2002.

[16] L. Hamel, Knowledge Discovery with Support Vector Machines, D. T. Larose, Ed. New Jersey: Wiley, 2009, pp. 171-183.

[17] H. Hormozi, E. Hormozi, and H. R. Nohoo, "The classification of the applicable machine learning methods in robot manipulators," International Journal of Machine Learning and Computing, vol.2, pp. 560-563, Oct. 2012.

[18] C. Cortes and V. Vapnik, "Support Vector Networks," Machine Learning, vol. 20, pp. 273-297, 1995.
[19] V. Kecman, "Support Vector Machines: An Introduction" in Support Vector Machines: Theory and Applications, L. Wang, Ed. Berlin: Springer-Verlag, 2005, ch.1, pp. 1-47.

[20] N. Cristianini and J. Shawe-Taylor, An Introduction to Support Vector Machines and Other Kernel-Based Learning Methods, 1st ed. Cambridge, U.K.: Cambridge University Press, 2000, pp. 93-124.

[21] V. Vapnik, Statistical Learning Theory, New York: Wiley, 1998.

[22] E. Osuna, R. Freund, and F. Girosi, Support Vector Machines: Training and Application, A.I. Memo No. 1602, Artificial Intelligence Laboratory, MIT, 1997.

[23] T. Y. Chen, "Comparative analysis of SAW and TOPSIS based on interval-valued fuzzy sets: Discussions on score functions and weight constraints," Expert Systems with Applications, vol. 39, pp. 1848-1861, Feb. 2012.

[24] H. B. Kartal, "Inventory Classification Using Machine Learning Methods and An Application of Support Vector Machines," M.Sc. Thesis, Department of Management Eng., Istanbul Technical Uni., Istanbul, Turkey, 2012

[25] N. Guvenir, "Application to Multi-criteria Inventory Classification," M.Sc. Thesis, Bilket University, Ankara, Turkey, 1993.

[26] N. Aissaoui, M. Haouari, and E. Hassini, "Supplier selection and order lot sizing modeling: A review," Computers \& Operations Research, vol. 34, pp. 3516-3540, 2007.

[27] J. Sarai, "Practical approach to the ABC analysis, its extension and application," Int. Sym. on Inventories, Budapest, pp. 255-261, 1980.

[28] V. Schmid, K. F. Doerner, and G. Laporte. (2013). "Rich routing problems arising in supply chain management," European Journal of Operational Research, vol. 224, pp. 435-448. [Online]. Available: http://www.sciencedirect.com/science/article/pii/S03772217120063 76

[29] D. T. Luc, "Pareto Optimality" in Pareto Optimality, Game Theory and Equilibria, A. Chinchuluun, M. Panos Pardalos, A. Migdalas, and L. Pitsoulis, Eds. New York: Springer, 2008, vol. 17, pp. 481-515.

[30] M. Hall, E. Frank, G. Holmes, B. Pfahringer, P. Reutemann, and I. H. Witten (June 2009); The WEKA data mining software: An update; SIGKDD Explorations. vol. 11, pp. 10-18. [Online]. Available: http://dl.acm.org/citation.cfm?id $=1656278$

[31] A. H. Wahbeh, Q. A. Radaideh, M. N. Al-Kabi, and E. M. Al-Shawakfa, "A comparison study between data mining tools over some classification methods," IJACSA, vol. Special issue on Artificial Intelligence, pp. 18-26, 2011.

[32] J. Platt. (January, 1998). Fast training of support vector machines using sequential minimal optimization. Advances in Kernel Methods -Support Vector Learning, MIT Press. pp. 41-65. [Online]. Available: http://research.microsoft.com/apps/pubs/?id=68391

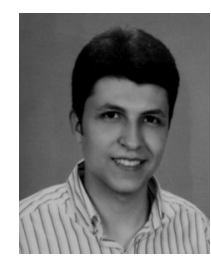

Hasan Basri Kartal is currently a PhD student in Industrial Engineering at Istanbul Technical University (ITU). He received his MSc degree in Management Engineering from ITU (2012) and earned his BSc in Industrial Engineering at Yildiz Technical University, Istanbul (2009)

He worked at Karadeniz Technical University as a Research Assistant in operational research area (2012) and in Ortadogu Energy (2010) as a part-time engineer. He also worked in Arcelik Electronics (2009) as a project based intern. And, he was an information system intern in Atmaca Household Appliances (2008), and a production systems intern in Senur Motors (2007) in Istanbul.

Mr. Kartal was a member of Turkish Chamber of Mechanical Engineers. He worked on lean manufacturing applications in Turkish textile industry. His current research interests are Machine Learning and its applications in industrial engineering related areas.

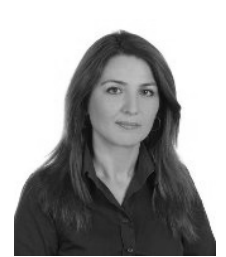

Dr. Ferhan Cebi is an Associate Professor of Production and Operations Management in the Faculty of Management at Istanbul Technical University (ITU). She holds a B.S. in Chemical Engineering from ITU (1985), a M.S. in Management Engineering from ITU (June 1989), and a Ph.D. in Management Engineering (2007) from the same university.

She gives the lectures on Operations Research and Operations Management at the undergraduate level and graduate level. Her main research areas are mathematical modeling in production and service sectors and competitiveness usage of information technology. Her works have been published in international and national journals and conference proceedings. 